\title{
Monte Carlo investigations of megavoltage cone-beam CT using thick, segmented scintillating detectors for soft tissue visualization
}

\author{
Yi Wang, ${ }^{\text {a) }}$ Larry E. Antonuk, Youcef El-Mohri, Qihua Zhao, Amit Sawant, and Hong Du \\ Department of Radiation Oncology, University of Michigan, Ann Arbor, Michigan 48109
}

(Received 5 June 2007; revised 7 November 2007; accepted for publication 7 November 2007; published 19 December 2007)

\begin{abstract}
Megavoltage cone-beam computed tomography (MV CBCT) is a highly promising technique for providing volumetric patient position information in the radiation treatment room. Such information has the potential to greatly assist in registering the patient to the planned treatment position, helping to ensure accurate delivery of the high energy therapy beam to the tumor volume while sparing the surrounding normal tissues. Presently, CBCT systems using conventional MV active matrix flatpanel imagers (AMFPIs), which are commonly used in portal imaging, require a relatively large amount of dose to create images that are clinically useful. This is due to the fact that the phosphor screen detector employed in conventional MV AMFPIs utilizes only $\sim 2 \%$ of the incident radiation (for a $6 \mathrm{MV}$ x-ray spectrum). Fortunately, thick segmented scintillating detectors can overcome this limitation, and the first prototype imager has demonstrated highly promising performance for projection imaging at low doses. It is therefore of definite interest to examine the potential performance of such thick, segmented scintillating detectors for MV CBCT. In this study, Monte Carlo simulations of radiation energy deposition were used to examine reconstructed images of cylindrical CT contrast phantoms, embedded with tissue-equivalent objects. The phantoms were scanned at $6 \mathrm{MV}$ using segmented detectors having various design parameters (i.e., detector thickness as well as scintillator and septal wall materials). Due to constraints imposed by the nature of this study, the size of the phantoms was limited to $\sim 6 \mathrm{~cm}$. For such phantoms, the simulation results suggest that a $40 \mathrm{~mm}$ thick, segmented CsI detector with low density septal walls can delineate electron density differences of $\sim 2.3 \%$ and $1.3 \%$ at doses of 1.54 and 3.08 cGy, respectively. In addition, it was found that segmented detectors with greater thickness, higher density scintillator material, or lower density septal walls exhibit higher contrast-to-noise performance. Finally, the performance of various segmented detectors obtained at a relatively low dose (1.54 cGy) was compared with that of a phosphor screen similar to that employed in conventional MV AMFPIs. This comparison indicates that for a phosphor screen to achieve the same contrast-to-noise performance as the segmented detectors $\sim 18$ to 59 times more dose is required, depending on the configuration of the segmented detectors. (C) 2008 American Association of Physicists in Medicine. [DOI: 10.1118/1.2818957]
\end{abstract}

Key words: active matrix flat-panel imager, segmented scintillating detectors, megavoltage conebeam CT, soft tissue visualization, contrast-to-noise ratio

\section{INTRODUCTION}

Technical advances in radiation therapy, such as threedimensional conformal ${ }^{1}$ and intensity modulated radiation therapy, ${ }^{2}$ have created the possibility of delivering treatment beams ever more precisely, leading to increased dose to tumor volumes and significant dose reduction to surrounding normal tissues and critical structures. However, the successful execution of high precision radiation treatments relies on accurate patient set-up in the treatment room so as to ensure the dose is delivered as intended. ${ }^{3}$ For this reason, a variety of volumetric imaging techniques are under investigation to provide tumor volume visualization in the treatment room immediately before the treatment. ${ }^{4-17}$ One such technique that has been clinically implemented involves acquiring cone-beam computed tomography (CBCT) images with a kilovoltage $(\mathrm{kV}) \mathrm{x}$-ray source and a diagnostic active matrix flat-panel imager (AMFPI), both orthogonally mounted to the treatment gantry. ${ }^{5-11}$ This system can produce useful CT images at clinically acceptable doses (i.e., a few cGy, which is approximately equal to the dose for one portal image). ${ }^{8-10}$

Another CT imaging technique that is also under investigation for soft tissue visualization involves the use of the megavoltage (MV) therapy beam and an electronic portal imaging device (EPID) ${ }^{5-8,11,18-32}$ Although the intrinsic contrast of human anatomical structures is lower at MV energies compared to $\mathrm{kV}$ energies, it has been demonstrated that soft tissues can be visualized on MV CBCT images obtained using conventional, as well as investigational, imagers. ${ }^{8,24,30,33}$ In addition, MV CBCT can offer some advantages over the $\mathrm{kV}$ technique. For example, MV CBCT makes use of the therapy beam itself and of a MV AMFPI that has already been installed for portal imaging, without the need for additional equipment. ${ }^{34}$ Also, MV CBCT images can, in principle, be directly used for high accuracy dose calculation $^{16,22,35}$ and inhomogeneity corrections ${ }^{36}$. Furthermore, MV CBCT is almost free of the "streak artifacts" that can occur at $\mathrm{kV}$ energies when metal objects, such as dental 
fillings and hip prostheses, are present in the imaged volume. $^{21,37}$ Finally, MV CBCT is much less subject to scatter radiation, which can degrade the image quality of $\mathrm{kV}$ CBCT. $^{22}$

Despite these advantages, clinical implementation of MV CBCT is constrained by the relatively low quantum efficiency (i.e., less than $2 \%$ at $6 \mathrm{MV}$ ) of the phosphor screen + metal plate $\mathrm{x}$-ray detectors used in conventional megavoltage AMFPIs. ${ }^{38,39}$ As a result, the dose needed for soft tissue visualization with such conventional imagers is relatively high. For example, Groh et al. reported the use of 32 cGy to delineate $\sim 4 \%$ density differences. ${ }^{8}$ Also, Morin et al. reported using $14.4 \mathrm{cGy}$ to obtain pelvic images with visible soft tissues (e.g., prostate, rectum, fat, and muscle) ${ }^{22}$ and 9 MU to obtain pelvic images having sufficient soft tissue information to guide $3 \mathrm{D}$ positioning. ${ }^{23}$ If the x-ray detector quantum efficiency could be greatly improved, it is reasonable to expect that soft tissues could be visualized at considerably lower, clinically acceptable doses using megavoltage cone-beam (or fan-beam) CT. For this reason, high efficiency x-ray detectors have been widely investigated. For example, 1D linear, as well as arc, detectors incorporating thick xenon gas ionization chambers ${ }^{32,33,40}$ and thick crystalline scintillating detectors have been constructed and evaluated. ${ }^{41-45}$ Moreover, a 2D matrix of $10 \mathrm{~mm}$ thick crystalline $\mathrm{CsI}(\mathrm{Tl})$ scintillating detector coupled with a TV camera has been examined. ${ }^{46,47}$ In addition, an AMFPI-based system incorporating a 2D matrix of $8 \mathrm{~mm}$ thick $\mathrm{CsI}(\mathrm{Tl})$ crystals (but with a much finer pitch and larger area than the camera system) has been developed $^{25}$ and recently used for lung tumor imaging and respiratory gating. ${ }^{26-28}$ Also, the feasibility of using thick optical fibers to detect Cerenkov radiation has been discussed. ${ }^{48}$ An alternative means for improving MV CBCT performance is to modify the treatment beam so as to generate lower energy $\mathrm{x}$ rays for imaging. This is currently under investigation through replacement of the usual tungsten target with a target made of low $Z$ material (e.g., carbon) and removing the flattening filter during image acquisition. ${ }^{49}$

Toward greatly increasing the x-ray detector efficiency of megavoltage imagers, initial theoretical and empirical investigations of up to $40 \mathrm{~mm}$ thick, segmented crystalline scintillating detectors have recently been reported by our group. ${ }^{50,51}$ A prototype AMFPI incorporating such a detector design has been constructed and has demonstrated highly promising performance at low doses. ${ }^{51}$ These encouraging early results motivate the present investigation of the potential performance of such detector designs for MV CBCT.

In this article, Monte Carlo methods simulating x-ray energy deposition are used to theoretically examine the contrast, noise, and contrast-to-noise performance of MV CBCT systems incorporating various segmented scintillating detector designs. The resulting performance is then compared to that from a simulated MV CBCT system employing a phosphor screen detector. The simulations suggest that soft tissue visualization can be achieved in MV CBCT images at clinically acceptable doses. Finally, the prospects for realization of optimized detector configurations for low dose MV CBCT are discussed.

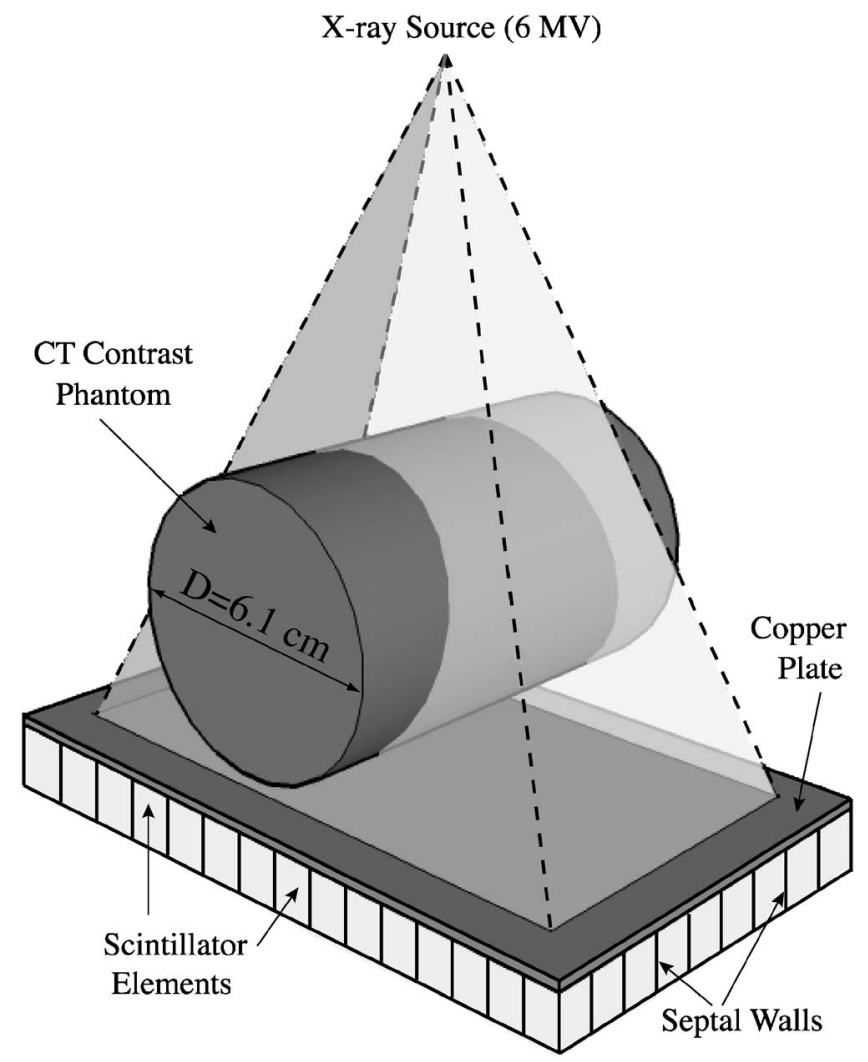

FIG. 1. Three-dimensional schematic view of the simulated, megavoltage cone-beam CT (MV CBCT) system.

\section{METHODS}

In order to quantify the performance of MV CBCT systems incorporating a variety of hypothetical segmented scintillating detector configurations, Monte Carlo simulation of energy deposited by the incident radiation was employed. (optical transport simulation was beyond the scope of this study.)

\section{II.A. Simulation set-up}

Figure 1 illustrates the simulated MV CBCT system. A point $\mathrm{x}$-ray source and a segmented scintillating detector were used to scan a cylindrical CT contrast phantom tomographically. The x-ray source employed a $6 \mathrm{MV}$ photon beam representing a typical spectral output along the central axis of a Varian linear accelerator. ${ }^{52}$ Any changes in the spectral output across the radiation field caused by the shape of the accelerator's flattening filter were not considered in the present simulations. However, for the small field size used in the study, these changes are minimal ${ }^{53}$ and would not significantly affect the results of the simulation. The center of the phantom and the entrance surface of the detector were 126 and $130 \mathrm{~cm}$ from the source, respectively. The detector had an area of $9.14 \times 4.12 \mathrm{~cm}^{2}$. The beam size at the entrance of the detector was $8.46 \times 3.17 \mathrm{~cm}^{2}$, which covered the whole CT contrast phantom in the radial direction. The segmented scintillating detector consisted of a matrix of $180 \times 81$ scintillator elements separated by septal walls and a $1 \mathrm{~mm}$ thick 

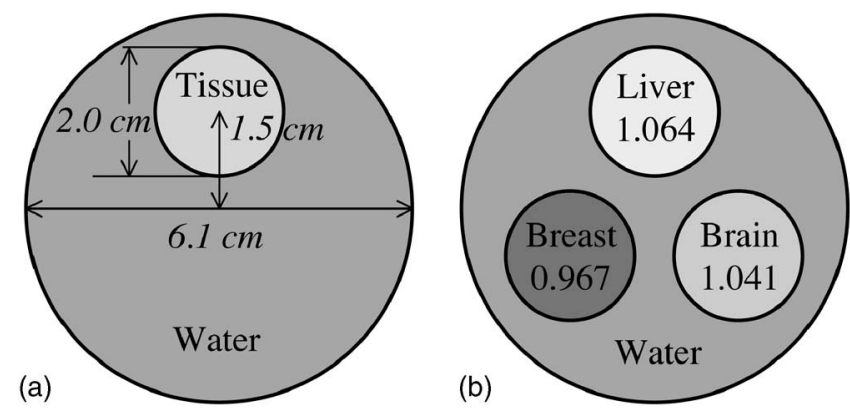

FIG. 2. Cross-sectional view of the cylindrical CT contrast phantoms simulated in this study. (a) Illustration of a phantom with a single tissueequivalent object. This phantom, using various tissue-equivalent objects, was employed in the performance evaluation of a $40 \mathrm{~mm}$ thick, segmented CsI detector with low density septal walls. (b) Illustration of a phantom with three specific tissue-equivalent objects. The electron densities of these objects relative to water are indicated in the figure. This phantom was employed in the performance evaluation of all $\mathrm{x}$-ray detectors examined in this study.

copper plate coupled to the x-ray side of the scintillator matrix acting as a radiation build-up layer. In this study, 10 and $40 \mathrm{~mm}$ thick, segmented CsI and BGO detectors, separated with low density (polystyrene), equivalent density (scintillator material), and high density (tungsten) septal walls were examined, for a total of 12 detector configurations. The detector element-to-element pitch was chosen to be $0.508 \mathrm{~mm}$, equal to the pixel pitch of an indirect-detection MV AMFPI array $^{54}$ that has been used in the empirical evaluation of a recent prototype.$^{51}$ The width of the septal walls was chosen to be $0.05 \mathrm{~mm}$, corresponding to the minimum value that can be achieved with present fabrication techniques. In the simulations, the energy deposited in the septal walls was not considered to contribute to the imaging signal, even in the cases where the septal walls were assumed to be the same material as the scintillator. The use of the three septal wall types allowed the examination of detector performance when the scintillator and the septal walls have greatly different, as well as similar, radiation attenuation properties. Ideally, the scintillator elements should be focused toward the $\mathrm{x}$-ray source ${ }^{50}$ so as to reduce the loss of spatial resolution induced by the $\mathrm{x}$ rays incident at oblique angles. For the present study however, due to the large source-to-detector distance and the limited detector size, the maximum incident angle of the $\mathrm{x}$ rays is only $\sim 2^{\circ}$. Therefore, the simulated system does not suffer greatly from the effect of oblique incidence of radiation, and thus, for reasons of simplicity, a nonfocused geometry was used.

For comparison, CBCT simulations were also performed with a conventional MV AMFPI incorporating a phosphor screen detector, which consisted of a $\mathrm{Gd}_{2} \mathrm{O}_{2} \mathrm{~S}: \mathrm{Tb}$ screen (surface density of $133 \mathrm{mg} / \mathrm{cm}^{2}$ ) and an overlying $1 \mathrm{~mm}$ thick copper plate. ${ }^{39}$ The screen had the same area as the segmented scintillators and was considered to consist of $180 \times 81$ voxels, with a voxel pitch of $0.508 \mathrm{~mm}$. The energy deposited in each voxel was taken as the imaging signal.

Figure 2 schematically illustrates the simulated CT contrast phantoms, each of which consists of one or three cylindrical tissue-equivalent objects embedded in a large "background" water cylinder. The diameters of the object cylinders and the large water cylinder were 2.03 and $6.10 \mathrm{~cm}$, respectively. The center of each object cylinder was positioned 1.52 $\mathrm{cm}$ from the center of the water cylinder. The length of all phantoms was $4.06 \mathrm{~cm}$. In this study, due to a limitation on the maximum number of array elements allowed by the simulation codes, it was not feasible to use segmented detectors with an element pitch of $0.508 \mathrm{~mm}$ to perform tomographic scans of CT contrast phantoms of human dimensions (e.g., 20 to $30 \mathrm{~cm}$ ). Figure 2(a) illustrates a phantom with a single embedded object. This phantom, which was used in six sets of simulations involving six tissue-equivalent materials, was scanned with a $40 \mathrm{~mm}$ thick, segmented CsI detector with low density septal walls. The chemical compositions and physical properties of these materials are summarized in Table I. Figure 2(b) shows a phantom with three objects, which was scanned with the various x-ray detectors. The three tissue-equivalent objects corresponded to a liver, a brain, and a breast, whose properties appear in Table I. The use of the three-object phantom to perform simulations involving these three materials considerably reduced the total amount of computational time required for this part of the study.

\section{II.B. Monte Carlo simulations}

EGSnrc $^{57}$ and DOSXYZnrc ${ }^{58}$ Monte Carlo codes, were used to simulate radiation energy deposition in various $\mathrm{X}$-ray detectors. In the simulations, the parameters of PCUT and

TABLE I. Chemical compositions by percentage of element weight, physical density, and relative electron density of the tissue-equivalent materials examined in this study.

\begin{tabular}{|c|c|c|c|c|c|c|c|c|c|c|c|c|}
\hline \multirow[b]{2}{*}{ Tissue-equivalent material } & \multicolumn{10}{|c|}{ Element weight $(\%)$} & \multirow{2}{*}{$\begin{array}{l}\text { Physical density } \\
\qquad\left(\mathrm{g} / \mathrm{cm}^{3}\right)\end{array}$} & \multirow[b]{2}{*}{ Relative electron density } \\
\hline & $\mathrm{H}$ & $\mathrm{C}$ & $\mathrm{N}$ & $\mathrm{O}$ & $\mathrm{Na}$ & $P$ & $S$ & $\mathrm{Cl}$ & K & $\mathrm{Ca}$ & & \\
\hline Breast $(\mathrm{BR} 12)^{\mathrm{a}}$ & 8.68 & 69.95 & 2.37 & 17.91 & & & & 0.14 & & 0.95 & 0.990 & 0.967 \\
\hline Lipoma $^{\mathrm{b}}$ & 10.90 & 71.90 & 3.00 & 13.80 & 0.10 & & 0.20 & 0.10 & & & 0.980 & 0.977 \\
\hline Mammary Gland (Adult \#1) & 10.90 & 50.60 & 2.30 & 35.80 & 0.10 & 0.10 & 0.10 & 0.10 & & & 0.990 & 0.987 \\
\hline Skin (Fetus) ${ }^{\mathrm{b}}$ & 10.80 & 5.10 & 1.20 & 82.10 & 0.20 & 0.10 & 0.10 & 0.30 & 0.10 & & 1.020 & 1.016 \\
\hline Brain $(\mathrm{SR} 2)^{\mathrm{a}}$ & 10.83 & 72.54 & 1.69 & 14.86 & & & & 0.08 & & & 1.045 & 1.041 \\
\hline Liver $(\operatorname{mix})^{\mathrm{a}}$ & 8.09 & 67.00 & 2.47 & 19.99 & & & & 0.14 & & 2.31 & 1.095 & 1.064 \\
\hline
\end{tabular}

${ }^{\mathrm{a}}$ Material information obtained from Ref. 55.

${ }^{\mathrm{b}}$ Material information obtained from Ref. 56. 
ECUT (corresponding to cutoff energies for photons and electrons) were chosen to be 0.01 and $0.521 \mathrm{MeV}$ (corresponding to a kinetic energy of $0.01 \mathrm{MeV}$ ), respectively. The EXACT boundary crossing algorithm, PRESTA-II electronstep algorithm, and NIST bremsstrahlung cross sections were used. The simulations were performed using a total of 264 CPUs on two 64-bit Linux clusters with 1.8 and $2.2 \mathrm{GHz}$ AMD Opteron processors, respectively, as well as a 64-bit Apple cluster with $2.0 \mathrm{GHz}$ G5 processors. This study required a total of $\sim 700000 \mathrm{CPU}$ hours.

\section{II.B.1. X-ray fluence to dose correspondence}

In this study, the amount of radiation used in the CBCT simulations was quantified in terms of the $\mathrm{x}$-ray fluence (i.e., the number of $\mathrm{x}$ rays per unit area) incident on the phantom. In order to allow a comparison of the irradiation conditions used in the simulations to those reported in other publications concerning MV CBCT, the equivalence between the $\mathrm{x}$-ray fluence and the irradiation time [in monitor units (MU)] for a therapy machine was established through a calibration simulation of the absorbed dose (in cGy) at a depth of $10 \mathrm{~cm}$, in a $30 \times 30 \times 30 \mathrm{~cm}^{3}$ water phantom. The surface of the water phantom was positioned $100 \mathrm{~cm}$ from the source, and the field size at the surface was $10 \times 10 \mathrm{~cm}^{2}$. Under such calibration conditions and for an irradiation time of $1 \mathrm{MU}$, a medical linac is typically calibrated so as to deposit $\sim 0.8$ to $1 \mathrm{cGy}$ of the dose at $6 \mathrm{MV}$. Therefore, the doses reported in this article correspond to the irradiation time (in monitor units) for therapy machines so calibrated, and thus represent equivalent calibration doses. (For simplicity, in this article, such equivalent calibration doses will be referred to, simply, as doses.) The calibration simulation was performed using $20 \times 10^{9} \mathrm{x}$ rays crossing the $10 \times 10 \mathrm{~cm}^{2}$ field at $100 \mathrm{~cm}$ and yielded a dose of $\sim 0.09 \mathrm{cGy}$, with a statistical uncertainty of $\sim 0.6 \%$. The x-ray fluence used in this calibration simulation corresponds to a value for 3.17 $\times 10^{9} \mathrm{x}$ rays over the $8.46 \times 3.17 \mathrm{~cm}^{2}$ field at $130 \mathrm{~cm}$ (where the CBCT simulations were performed). Thereafter, for a given CBCT simulation using $N$ x rays, the corresponding dose is given by

$$
\text { Dose }=\frac{N}{3.17 \times 10^{9}} \times 0.09(\mathrm{cGy}) .
$$

For example, for the simulations involving the segmented scintillating detectors, $0.6 \times 10^{9} \mathrm{x}$ rays were used for each of 180 projection images, resulting in a total scan dose of 3.08 cGy. For the simulations involving the phosphor screen detector, $19.8 \times 10^{9} \mathrm{x}$ rays were used per projection, resulting in a total scan dose of $101.64 \mathrm{cGy}$.

\section{II.B.2. Cupping artifact removal}

A cupping artifact, arising from radiation scattering, ${ }^{9,59,60}$ was observed on the reconstructed images. This artifact was manifested as a general increase in signal along the radial direction from the center to the edge of the cylindrical CT phantom. A standard method to remove this artifact involves determination and subtraction of correction factors based on the averaged radial signal in the reconstructed image of a uniform water phantom having the same dimensions as the contrast phantom and obtained using the same simulation set-up. ${ }^{61}$ However, this method was impractical to implement for all detector configurations studied. Since each configuration required its own correction, which consumes as much computational time as scanning the contrast phantom, an additional $\sim 500000$ CPU hours would have been required for all configurations. This was beyond the resources available to the study. For that reason, a simplified determination method was developed. Instead of simulating an additional uniform water phantom, the simplified method involved the use of the water region in the reconstructed image of the contrast phantom itself to obtain the correction factors. For validation purposes, both methods were examined and compared for the case of a $40 \mathrm{~mm}$ thick, segmented CsI detector with low density septal walls.

\section{II.B.3. Performance evaluation}

The Monte Carlo investigations presented in this article consist of the evaluation of MV CBCT systems incorporating various segmented scintillating detectors and a phosphor screen detector. A $40 \mathrm{~mm}$ thick, segmented CsI detector with low density septal walls was chosen to examine system performance as a function of slice thickness ranging from 1 to $20 \mathrm{~mm}$, as well as for total scan doses ranging from 1.54 to $6.16 \mathrm{cGy}$. The same detector was also used to study a variety of tissue-equivalent materials at 1.54 and 3.08 cGy. In addition, three tissue-equivalent materials (i.e., liver, brain, and breast) were chosen to be scanned using the various segmented detectors with different detector thicknesses, scintillator materials, and septal wall materials, at $3.08 \mathrm{cGy}$. The same three materials were also studied at much higher doses (up to $101.64 \mathrm{cGy}$ ) using the phosphor screen detector, the performance of which was compared with that of the segmented detectors at $1.54 \mathrm{cGy}$.

\section{II.C. Analysis methods}

Each CT contrast phantom was scanned over $360^{\circ}$, at $2^{\circ}$ intervals, resulting in a total of 180 projection images. A Feldkamp-based algorithm using a ramp filter, ${ }^{62}$ which employs attenuation line integrals $(l)$ along straight pathways from the source to the imager pixels, was used to reconstruct the spatial distribution of attenuation coefficients for the CT phantoms. At each projection angle, $l$ can be calculated using the exponential attenuation formula

$$
\frac{I_{1}}{I_{0}}=e^{-l} \Rightarrow l=\ln \frac{I_{0}}{I_{1}}
$$

where $I_{1}$ and $I_{0}$ are x-ray intensity signals obtained from projection images with and without the phantom, respectively. In order to minimize the statistical error induced by $I_{0}$, 30 flood-field images (i.e., images acquired without a phantom), each obtained with the same dose as that used for a phantom image, were averaged. For each scan, one averaged flood-field image was used for all projection angles. The reconstructed voxel pitch and single slice thickness were cho- 


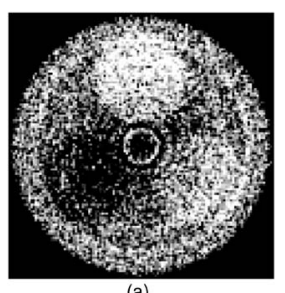

(a)

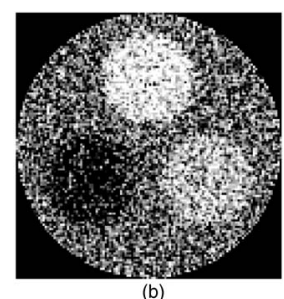

(b)
FIG. 3. Reconstructed images of a three-object CT contrast phantom (a) before and (b) after the correction for a cupping artifact using the standard method (described in the text). The results were obtained using the $40 \mathrm{~mm}$ thick, segmented CsI detector with low density septal walls. The orientation of the objects in the phantom is the same as that in Fig. 2(b). Note that the same window level was selected for both images.

sen to be $0.508 \mathrm{~mm}$, matching the pixel pitch of the AMFPI array. ${ }^{54}$ From the reconstructed image, a number of single slices were averaged to obtain a slice image with better statistics (e.g., $5.08 \mathrm{~mm}$ for ten slices).

In the reconstructed images, the mean signals in the water background $\left(\mu_{\text {water }}\right)$ and in the object $\left(\mu_{\text {obj }}\right)$ were extracted to evaluate performance. The selection of the regions from which data were extracted excluded the edges of the objects and the phantom as well as the center of the phantom where the cupping artifact correction induced non-negligible statistical uncertainties [see Eq. (A5) in the Appendix].

The deduced signal and noise are expressed in units of MVCT number, assuming a value of 0 Hounsfield units (HU) in water, and $-1000 \mathrm{HU}$ in air (or vacuum). Metrics evaluated in this study include contrast (Contrast), noise (Noise), and contrast-to-noise ratio $(\mathrm{CNR})$ of the tissue-equivalent objects in the reconstructed images. The Contrast of the object can be expressed as

$$
\text { Contrast }=\frac{\left|\mu_{\mathrm{obj}}-\mu_{\mathrm{water}}\right|}{\mu_{\mathrm{water}}} \times 1000(\mathrm{HU}) .
$$

Similarly, the Noise in the object can be expressed as

$$
\text { Noise }=\frac{\sigma_{\text {obj }}}{\mu_{\text {water }}} \times 1000(\mathrm{HU}),
$$

where $\sigma_{\mathrm{obj}}$ is the standard deviation of the voxel signal in the object. Therefore, the CNR of the object can be expressed as

$$
\mathrm{CNR}=\frac{\text { Contrast }}{\text { Noise }}=\frac{\left|\mu_{\mathrm{obj}}-\mu_{\text {water }}\right|}{\sigma_{\mathrm{obj}}} .
$$

The statistical uncertainties in the performance metrics were examined using a previously described method for CT imaging. ${ }^{63}$ The details of the statistical uncertainty analysis can be found in the Appendix.

\section{RESULTS}

\section{III.A. Cupping artifact removal}

Figure 3 shows reconstructed images of a three-object phantom [as illustrated in Fig. 2(b)] before and after the application of the standard method for cupping artifact removal. The reconstructed images and the correction factors

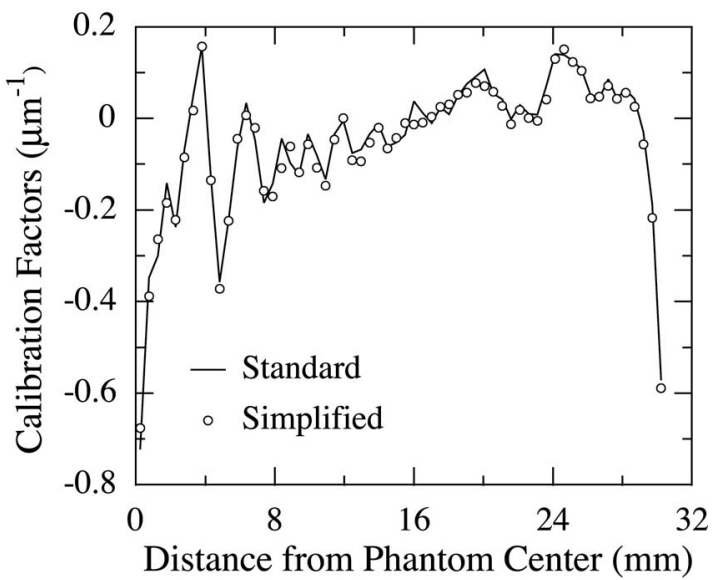

FIG. 4. Comparison of cupping artifact correction factors obtained with the standard method and with the simplified method (described in the text). The results were obtained using the same detector as that used in Fig. 3. The factors are illustrated as a function of the radial distance from the center of the phantom.

for cupping artifact removal were obtained using a $40 \mathrm{~mm}$ thick, segmented CsI detector with low density septal walls. Since the standard method requires the acquisition of independent CT scans with a uniform water cylinder so as to obtain correction factors, it was not practical to apply to all detector configurations. Instead, a simplified method based on acquiring the correction factors from the CT phantom images themselves was employed. In order to validate this simplified method, the correction factors obtained using this method were compared to those obtained using the standard method, as illustrated in Fig. 4. The factors obtained using both methods are very similar, indicating that they are equally effective in removing the cupping artifact. Moreover, the CNR results calculated from images corrected with both methods differed by only $\sim 2 \%$, which is much lower than the $\sim 7 \%$ statistical error in the CNR results. Therefore, the simplified method can be used as a valid estimation for removing the cupping artifact. In the remainder of this section the simplified method will be applied unless otherwise stated.

\section{III.B. Performance evaluation}

\section{III.B.1. Slice thickness and total scan dose}

The $40 \mathrm{~mm}$ thick, segmented CsI detector with low density septal walls was selected to demonstrate the square of $\mathrm{CNR}\left(\mathrm{CNR}^{2}\right)$ performance as a function of the slice thickness and total scan dose. Figure 5(a) illustrates $\mathrm{CNR}^{2}$ performance as a function of slice thickness (up to $20 \mathrm{~mm}$ ) at a total scan dose of $3.08 \mathrm{cGy}$. The $\mathrm{CNR}^{2}$ results are shown to exhibit a linear increase with increasing slice thickness. Figure 5(b) illustrates $\mathrm{CNR}^{2}$ performance as a function of total scan dose (up to $6.16 \mathrm{cGy}$ ) for a slice thickness of $5.08 \mathrm{~mm}$. These $\mathrm{CNR}^{2}$ results also exhibit a linear increase with increasing total scan dose. The linear behaviors observed in Figs. 5(a) and 5(b) are both due to the linear decrease in the square of Noise (Noise ${ }^{2}$ ) in the objects with increasing number of sampled quanta, resulting in improved statistics. In the 

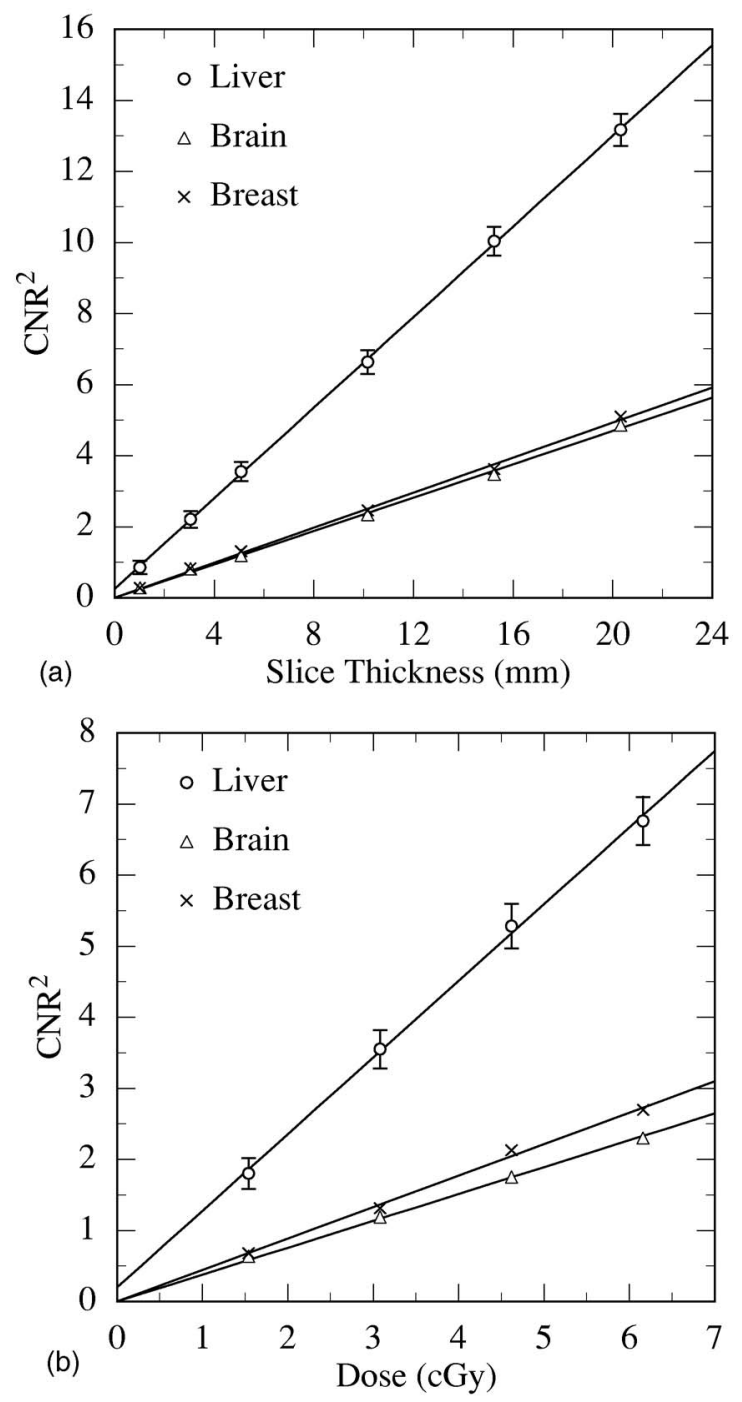

FIG. 5. CNR ${ }^{2}$ as a function of (a) reconstructed slice thickness and (b) total scan dose obtained using the $40 \mathrm{~mm}$ thick, segmented CsI detector with low density septal walls. The solid lines indicate linear fits to the data. For clarity of presentation, error bars are shown only for the liver-equivalent object. For a given slice thickness or dose, the relative errors in $\mathrm{CNR}^{2}$ for the other two objects are approximately the same as that for the liver-equivalent object. Note that in this figure and in the remaining figures, the label "dose" refers to the equivalent calibration dose as defined in Sec. II B 1 .

remainder of the Results section, performance metrics will be examined with a $5.08 \mathrm{~mm}$ thick reconstructed slice (obtained by averaging ten consecutive individual slices), and the segmented detectors will be examined at 1.54 and 3.08 cGy.

\section{III.B.2. Tissue-equivalent objects}

The CT contrast phantoms embedded with various tissueequivalent objects [as illustrated in Fig. 2(a)] were examined using the $40 \mathrm{~mm}$ thick, segmented CsI detector with low density septal walls. In this case, the effect of the cupping artifact was removed using the standard method. Figure 6(a) illustrates results for Contrast at $3.08 \mathrm{cGy}$ and Noise at 1.54 and $3.08 \mathrm{cGy}$, plotted as a function of the relative electron density of the various tissue-equivalent objects. The Contrast
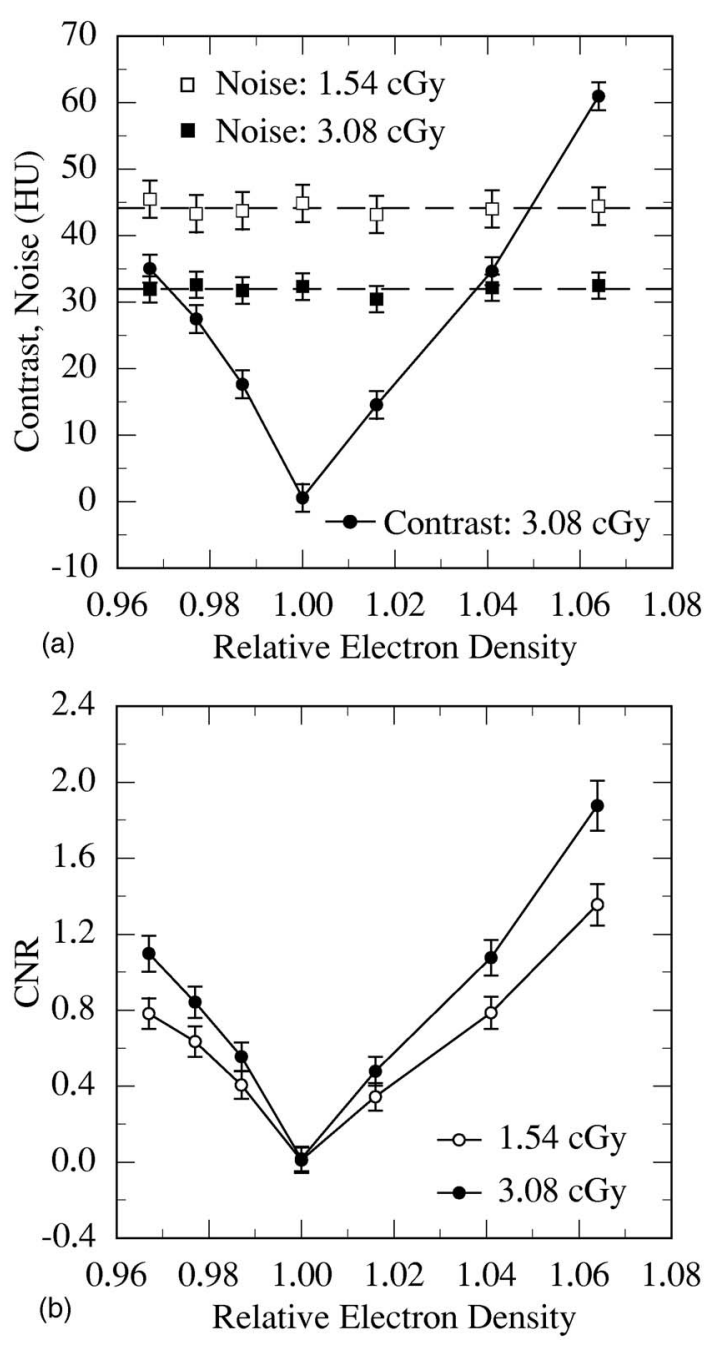

FIG. 6. Simulation results plotted as a function of the relative electron density of the various tissue-equivalent objects examined in this study. (a) Contrast at $3.08 \mathrm{cGy}$, as well as Noise results at 1.54 and $3.08 \mathrm{cGy}$. The solid line joining the Contrast data points is drawn to guide the eye, whereas the dashed lines indicate the mean levels of the Noise results at two doses. (b) CNR at 1.54 and $3.08 \mathrm{cGy}$. The solid lines joining the CNR data points are drawn to guide the eye.

results at $1.54 \mathrm{cGy}$ are almost the same as those at $3.08 \mathrm{cGy}$ and are not plotted for reasons of clarity of presentation. For the low contrast tissue-equivalent objects that have been examined, the Contrast results increase approximately linearly with increasing difference in relative electron density between the objects and the water background, due to the dominant effect of Compton scattering at megavoltage energies. The slight deviations from linear behavior are due to the presence of lower probability interactions caused by pair production, whose probability is not proportional to the electron density. In addition, it is found that within the range of statistical uncertainty, the Noise performance is independent of the relative electron density for the tissue-equivalent objects studied. The reduced level of Noise for the higher dose is due to the larger number of $x$ rays sampled. Figure 6(b) illustrates the CNR performance of the tissue-equivalent objects. The CNR results increase in a pattern similar to that 


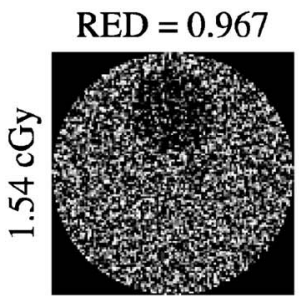

(a)

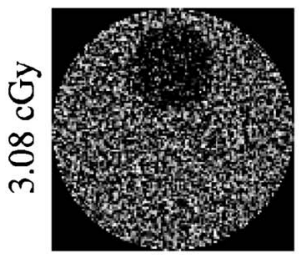

(g)

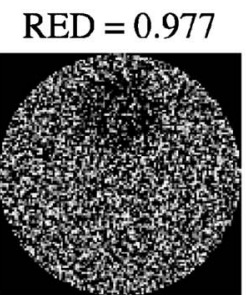

(b)

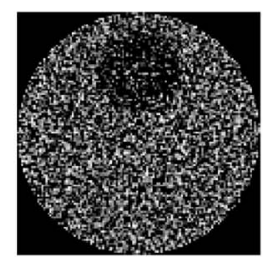

(h)

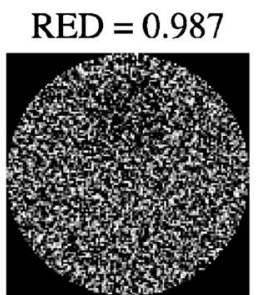

(c)

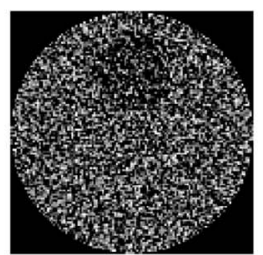

(i)

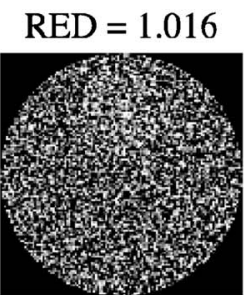

(d)

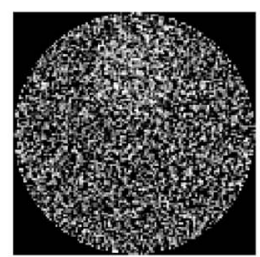

(j)

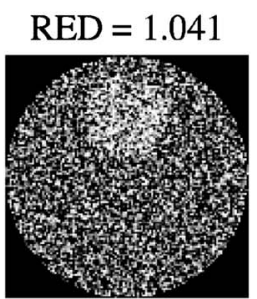

(e)

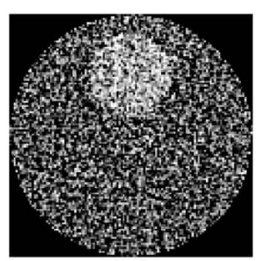

$(\mathrm{k})$

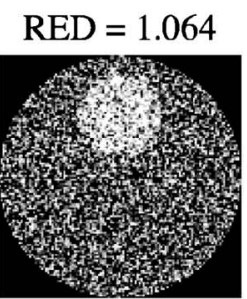

(f)

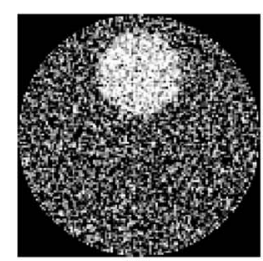

(I)

FIG. 7. Reconstructed images of the CT contrast phantoms embedded with various tissue-equivalent objects. For each phantom, a pair of images obtained at 1.54 and $3.08 \mathrm{cGy}$ is shown. The relative electron densities (RED) of the objects are indicated for each image pairs. The same window level was selected for the images obtained at the same dose, whereas different window levels were selected for different doses in order to maximize the visibility of the objects.

observed for the Contrast results. The improvement in CNR at the higher dose is due to a reduced level of Noise. Figure 7 shows the reconstructed images of the CT contrast phantoms incorporating the various objects at 1.54 and $3.08 \mathrm{cGy}$. As illustrated, it is possible for the segmented detector to delineate $2.3 \%$ [Fig. 7(b)] and 1.3\% [Fig. 7(i)] electron density differences at 1.54 and $3.08 \mathrm{cGy}$, respectively. Also, it is observed that the visibility of the tissue-equivalent objects improves with dose and with the relative electron density difference between each object and water, as expected and in line with the CNR results of Fig. 6(b).

\section{III.B.3. Segmented scintillating detector configurations}

Segmented scintillating detectors with different detector thicknesses, scintillator materials, and septal wall materials were examined at 3.08 cGy using the three-object CT contrast phantom [as illustrated in Fig. 2(b)]. Figure 8 illustrates Contrast, Noise, and CNR performance of the three tissueequivalent objects. The Contrast performance is mainly determined by the relative electron density of the object. It is observed that the thicker detectors exhibit a slightly lower Contrast, probably due to the increased relative contribution of phantom scatter radiation to detector signal. Since scatter radiation is lower in energy compared with the primary radiation, it is more efficiently absorbed as detector thickness increases. The effect of the scintillator and septal wall materials on Contrast is not pronounced. The Noise performance is mainly determined by the detector properties. In general, detectors with a greater thickness, higher density scintillator, and lower density septal walls, provide lower Noise. The Noise values of the three objects scanned with the same detector are observed to be equivalent within the range of statistical uncertainty. Generally, it is found that detectors with a greater thickness, higher density scintillator, and lower density septal walls offer higher CNR performance for a given object, mainly due to the reduced level of Noise.

Figure 9 shows $\mathrm{CNR}^{2}$ results for the liver-equivalent object obtained using segmented detectors with low and high density septal walls. These results, obtained at $3.08 \mathrm{cGy}$, are plotted as a function of the zero frequency detective quantum efficiency (DQE) of the corresponding detectors. These $\mathrm{DQE}$ values were reported in a previous Monte Carlo-based study. ${ }^{50}$ As shown in the figure, for detectors with the same septal wall density (i.e., low or high), $\mathrm{CNR}^{2}$ increases with increasing DQE. Moreover, it is found that for a given combination of detector thickness and scintillator material (e.g., $40 \mathrm{~mm}$ BGO) the detectors with higher density septal walls exhibit lower $\mathrm{CNR}^{2}$ performance, despite providing a higher DQE. This behavior is a consequence of the fact that higher density septal walls provide a higher degree of radiation attenuation. On the one hand, this higher attenuation results in a higher x-ray quantum efficiency, leading to better DQE performance. On the other hand, the higher attenuation also limits the lateral spread of the radiation and reduces the effect of statistical averaging, leading to a larger variation of the voxel signals in the reconstructed image (i.e., Noise $^{2}$ ) and thus lower $\mathrm{CNR}^{2}$ performance.

Figure 10 shows reconstructed images of the three-object phantom obtained using the various segmented scintillating detectors at $3.08 \mathrm{cGy}$. As illustrated, the three objects are clearly visible on all reconstructed images. In addition, the visibility of the objects improves with increasing detector thickness and scintillator density, and with decreasing septal wall density, in line with the CNR results of Fig. 8.

\section{III.B.4. Segmented detectors versus phosphor screen}

In this subsection, the performance obtained using the various segmented scintillating detectors is compared with that obtained using a phosphor screen detector, whose com- 

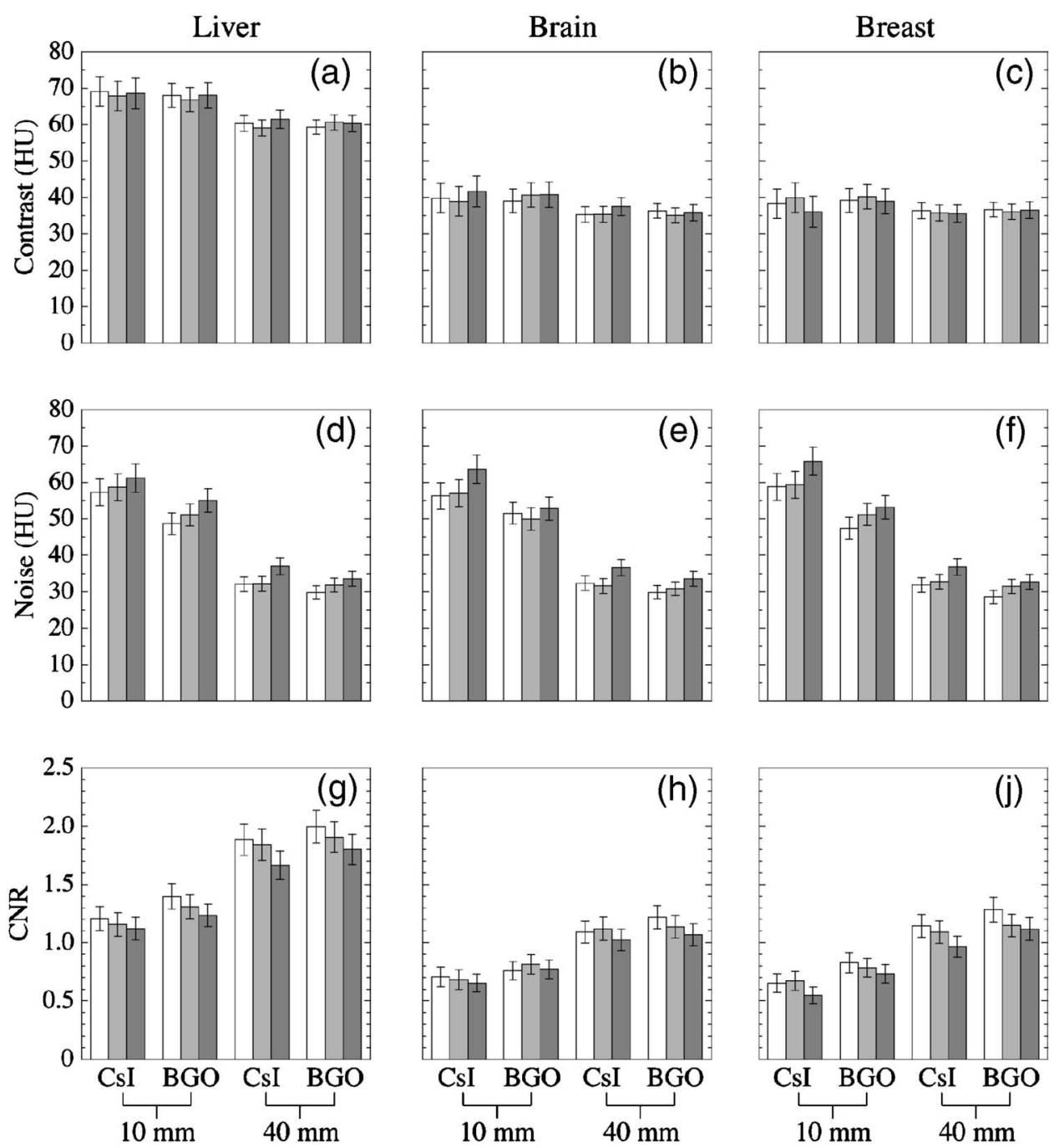

Septal Wall Density: $\square$ Low $\square$ Equivalent $\square$ High

FIG. 8. Contrast, Noise, and CNR results for the liver-, brain-, and breast-equivalent objects obtained using various segmented scintillating detectors. In each figure, the results obtained using the detectors with the same thickness and scintillator material are illustrated in one group. Moreover, in each group, detector designs with different septal wall materials are indicated by columns with different shadings.

position is representative of those used in conventional MV AMFPIs. The simulations were performed using the threeobject phantom. Figure 11 shows the $\mathrm{CNR}^{2}$ performance for the liver-equivalent object as a function of the total scan dose for the $40 \mathrm{~mm}$ thick, segmented CsI detector with low density septal walls. The results are compared with those obtained from the phosphor screen detector. The segmented detector exhibits impressive performance, with $\mathrm{CNR}^{2}$ increasing $\sim 49$ times faster with dose than with the phosphor screen detector (1.121 $\mathrm{cGy}^{-1}$ compared with $0.023 \mathrm{cGy}^{-1}$, respectively). Figures 12(a) and 12(b) show reconstructed images obtained at $4.62 \mathrm{cGy}$ using the same pair of detectors used in Fig. 11. All three objects are clearly visible in Fig. 12(a) (segmented detector), but are almost invisible in Fig. 12(b) (phosphor screen detector).

For the liver-equivalent object, the $\mathrm{CNR}^{2}$ performance of all segmented scintillating detectors is compared with that of the phosphor screen detector in Fig. 13. The phosphor screen detector performance (solid line) corresponds to the linear fit of the phosphor screen detector results in Fig. 11, and is illustrated as a function of dose (bottom $x$-axis scale). The plotted values of $\mathrm{CNR}^{2}$ obtained for the various segmented scintillating detectors at a constant dose of $1.54 \mathrm{cGy}$ (cross symbols) have been positioned along the $x$-axis direction so as to overlie the phosphor screen detector performance curve. With this plotting convention, the degree of dose reduction provided by the segmented detectors compared with the phosphor screen detector, for an equivalent level of $\mathrm{CNR}^{2}$ performance, is given by the top $x$-axis scale. For example, to achieve a $\mathrm{CNR}^{2}$ performance equivalent to that of the $40 \mathrm{~mm}$ thick, segmented BGO detector with low density septal walls at $1.54 \mathrm{cGy}$, the phosphor screen requires a dose of $\sim 90 \mathrm{cGy}$. Thus, if the phosphor screen were replaced by such a segmented detector, $\sim 59$ times less dose would be required to achieve equivalent $\mathrm{CNR}^{2}$ performance. 


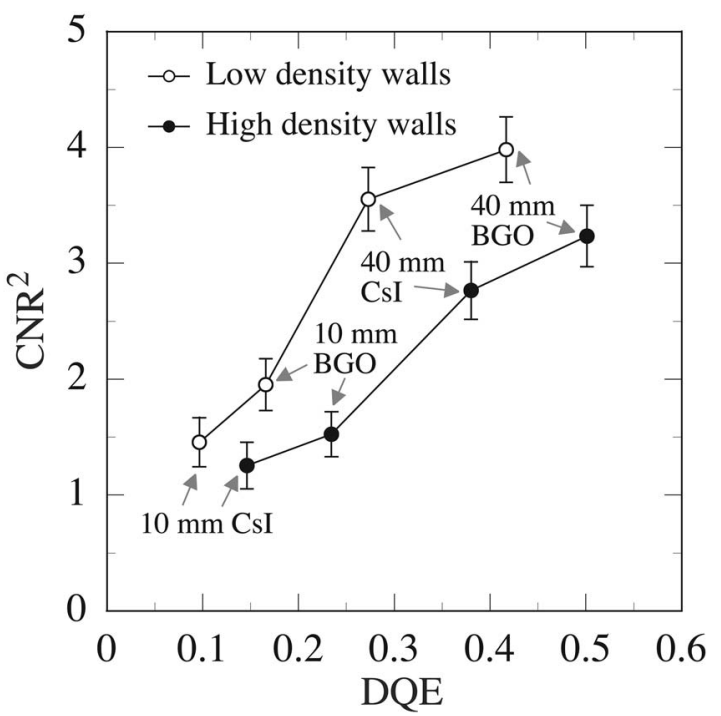

FIG. 9. CNR ${ }^{2}$ results for the liver-equivalent object. These results were obtained using the segmented detectors with low and high density septal walls. The data are plotted as a function of zero frequency DQE of the corresponding segmented scintillating detectors. The detector thickness and scintillator material are indicated for each detector. The lines joining the data points are drawn to guide the eye.

\section{DISCUSSION AND CONCLUSIONS}

In this article, a theoretical evaluation of the performance of thick, segmented scintillating detectors for the visualiza- tion of low contrast soft tissues using MV CBCT at clinically acceptable doses (e.g., $\sim 3$ cGy) for a 6 MV x-ray energy spectrum has been reported. Reconstructed images of a water phantom embedded with various tissue-equivalent objects were obtained through simulation of the energy deposited in the scintillating material. The Contrast, Noise, and CNR performances were examined as a function of slice thickness, total scan dose, relative electron density of the tissueequivalent materials, and various design parameters of the segmented scintillating detectors. The performance of various segmented detector configurations obtained at a relatively low dose ( $1.54 \mathrm{cGy})$ was compared to that of a phosphor screen detector, whose composition is representative of those used in conventional MV AMFPIs.

Within the limitations of this study (discussed below), the results indicate that the Contrast of a tissue-equivalent object is mainly determined by the difference in relative electron density between the tissue-equivalent object and the water background, due to the dominant effect of Compton scattering at megavoltage energies. The Contrast is not largely affected by the properties of the detector. In addition, for the low contrast tissue-equivalent objects that have been examined, and for a given detector, the Noise results appear to be independent of the relative electron density within the range of statistical uncertainty. The $\mathrm{CNR}^{2}$ performance for a tissueequivalent object exhibits an approximately linear increase with increasing slice thickness and scan dose, due to the

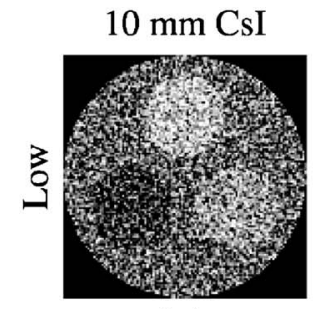

(a)

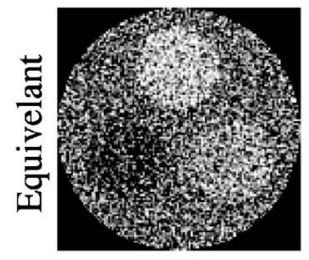

(e)

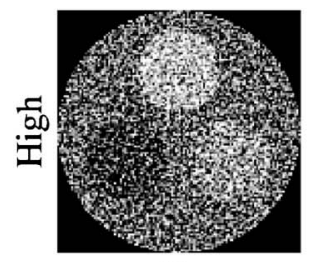

(i)

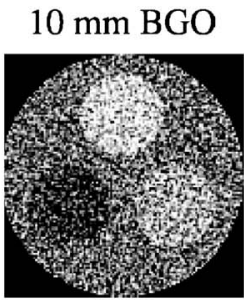

(b)

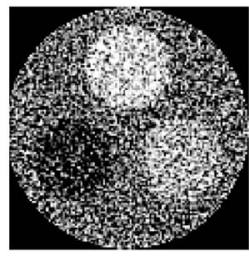

(f)

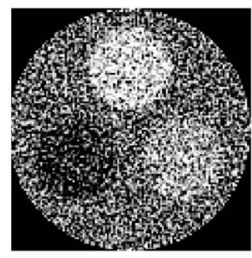

(j)

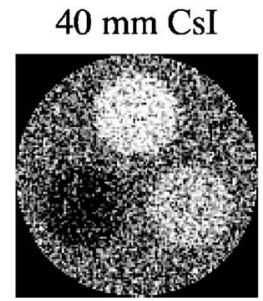

(c)

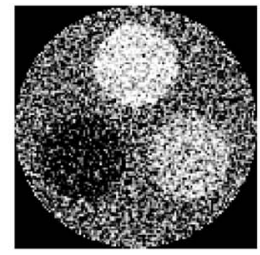

(g)

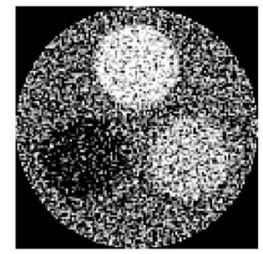

(k)

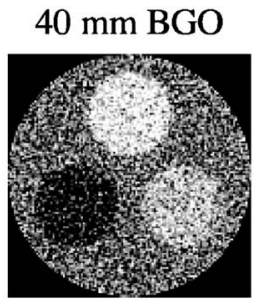

(d)

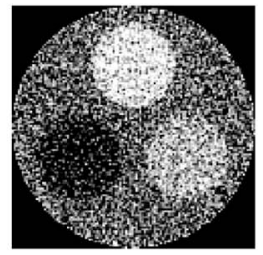

(h)

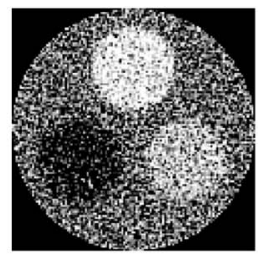

(I)

FIG. 10. Reconstructed images of the three-object phantom obtained using various segmented detectors at 3.08 cGy. Each column represents detectors with a given detector thickness and scintillator material, whereas each row represents detectors with a given type of septal wall density (i.e., low, equivalent, and high). The orientation of the objects in the phantom is the same as in Fig. 2(b). The same window level was selected for the images in the same column, while different window levels were selected for different columns to maximize the visibility of the objects. 


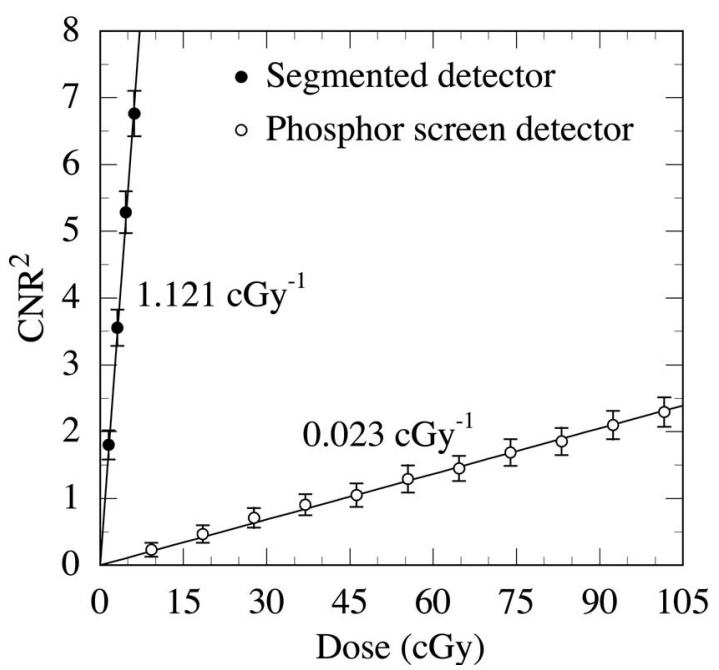

FIG. 11. $\mathrm{CNR}^{2}$ results for the liver-equivalent object as a function of total scan dose. Results are shown for the $40 \mathrm{~mm}$ thick, segmented CsI detector with low density septal walls. Results are also shown for a phosphor screen detector representative of the type used in conventional MV AMFPIs. The solid lines indicate linear fits to the data. The slope of the linear fit, representing the sensitivity of $\mathrm{CNR}^{2}$ to dose, is indicated for each detector.

reduced Noise ${ }^{2}$ values induced by the increasing number of $x$ rays sampled. In addition, with the same slice thickness and scan dose, the segmented detectors with greater thickness, higher density scintillators, or lower density septal walls result in lower Noise, and thus enhanced CNR performance. As seen from the reconstructed images, for the size of the phantoms studied, a $40 \mathrm{~mm}$ thick segmented CsI detector with low density septal walls allows resolution of electron density differences of $\sim 2.3 \%$ and $1.3 \%$ at 1.54 and 3.08 cGy, respectively. Moreover, all examined segmented detectors can delineate a 3.3\% electron density difference at 3.08 cGy. In addition, the results also indicate that for a phosphor screen detector to achieve the same $\mathrm{CNR}^{2}$ performance as the segmented detectors, $\sim 18$ to 59 times more dose is required, depending on the configuration of the segmented detector.

Due to constraints imposed by the simulation codes (see Sec. II A), the contrast phantoms examined were relatively small $(\sim 6 \mathrm{~cm}$ in diameter). For larger phantoms, there would be more radiation attenuation and scatter, which would be expected to degrade CNR performance, so that a higher dose would be required to maintain the same perfor-

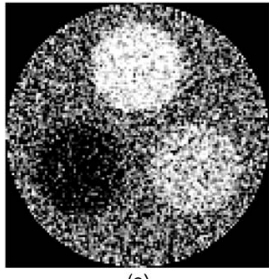

(a)

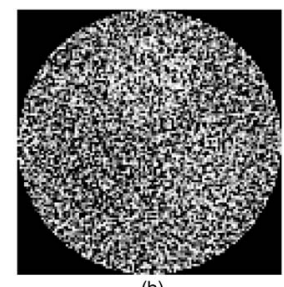

(b)

FIG. 12. Reconstructed images of the three-object phantom obtained at 4.62 cGy using (a) the $40 \mathrm{~mm}$ thick segmented CsI detector with low density septal walls and (b) the phosphor screen detector.

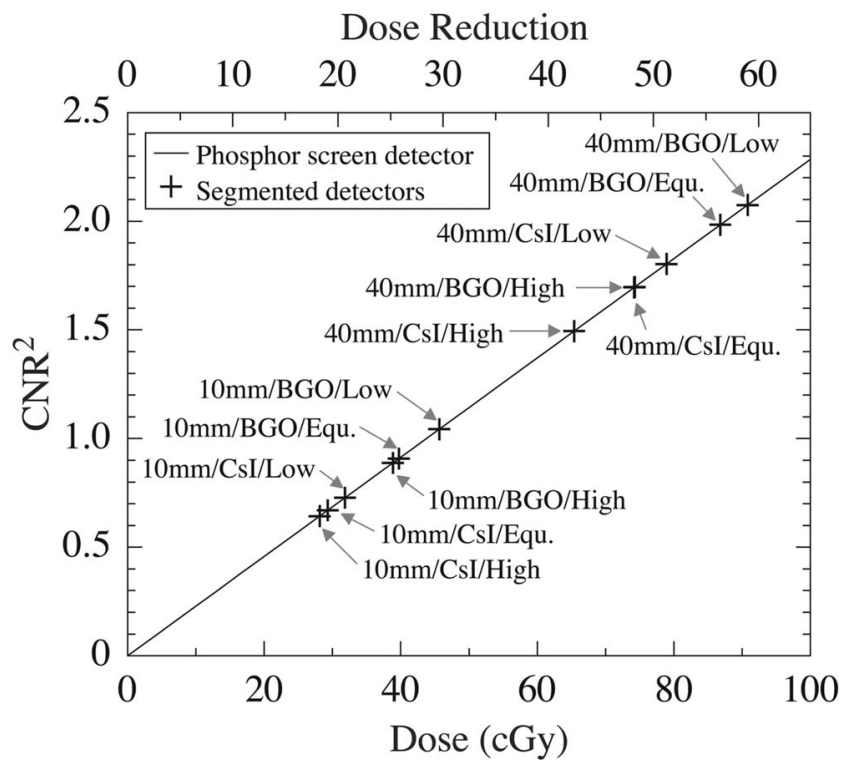

FIG. 13. Comparison of the $\mathrm{CNR}^{2}$ performance obtained using the various segmented detectors at $1.54 \mathrm{cGy}$ with that obtained using the phosphor screen detector at much higher doses. The solid line represents the $\mathrm{CNR}^{2}$ performance of the phosphor screen plotted as a function of dose (bottom $x$-axis scale). The cross symbols, which represent the $\mathrm{CNR}^{2}$ values obtained using the various segmented detectors at $1.54 \mathrm{cGy}$, are positioned along the $x$-axis direction so as to fall on the phosphor screen performance line; thus the degree of dose reduction offered by the segmented detectors compared to the phosphor screen is represented by the top $x$-axis scale. For each segmented detector result, the design parameters of the corresponding detector ("detector thickness/scintillator material/septal wall density") are indicated in the figure. Note that "low", "equ.", and "high" refer to the low, equivalent, and high density walls used in the simulations. Also note that the results for "40 mm/BGO/High" and "40 mm/CsI/Equ." almost exactly overlap.

mance obtained from the smaller phantoms. (Additional, exploratory simulations performed on simpler unsegmented scintillating detectors suggest that $\sim 3$ to 4 times more dose would be required to maintain the same CNR performance for a $20 \mathrm{~cm}$ phantom.) Nevertheless, it is suspected that, had a larger phantom been used, the CNR performance of the segmented detectors relative to the phosphor screen detector would not be significantly affected, since the degree of radiation attenuation and scattering is largely determined by the phantom.

In order to create images that are clinically useful, the CBCT system must have sufficient contrast-to-noise and spatial resolution performance. Table II lists the CNR values at 3.08 cGy obtained from this study and the modulation transfer function (MTF) values at 0.5 and 1 line pair/mm obtained for the same segmented detector configurations from previous Monte Carlo studies. ${ }^{50}$ For comparison, MTF values obtained from measurements with a conventional MV AMFPI, with a pixel pitch of $0.508 \mathrm{~mm}$ and employing a Lanex Fast B (Eastman Kodak) phosphor screen equivalent to that simulated in the present study, are also shown. ${ }^{38}$ The MTF values of the segmented detectors are all observed to be higher than that of the phosphor screen at both spatial frequencies, except for the case of the $40 \mathrm{~mm}$ thick, segmented CsI detector with low density septal walls at 0.5 line pair $/ \mathrm{mm}$. Note that 
TABLE II. For the various segmented detectors (columns 2-9) and the phosphor screen detector (column 10), CNR results for the liver-equivalent object at 3.08 cGy $\left(\mathrm{CNR}_{\mathrm{Liver}}\right)$ are shown. The CNR result for the phosphor screen detector was interpolated from the slope of $\mathrm{CNR}^{2}$ versus dose results illustrated in Fig. 11. Also shown are MTF results at 0.5 and 1.0 line pair/mm $\left(\mathrm{MTF}_{0.5}\right.$ and $\left.\mathrm{MTF}_{1.0}\right)$ obtained from a previous study of Monte Carlo simulations of radiation energy deposition involving the same segmented detector configurations (Ref. 48). For comparison, MTF results obtained from measurements (Ref. 36) with an MV AMFPI employing a phosphor screen (Lanex Fast B, Eastman Kodak) equivalent to that simulated in the present study are also listed in the last column. Note that, for the legend describing the segmented detectors, the first row indicates the detector thickness and scintillator material, and the second row indicates the septal wall density.

\begin{tabular}{lccccccccc}
\hline \hline & \multicolumn{2}{c}{$10 \mathrm{~mm} \mathrm{CsI}$} & \multicolumn{2}{c}{$40 \mathrm{~mm} \mathrm{CsI}$} & \multicolumn{2}{c}{$10 \mathrm{~mm} \mathrm{BGO}$} & \multicolumn{2}{c}{$40 \mathrm{~mm}$ BGO } \\
\cline { 2 - 8 } & Low & High & Low & High & Low & High & Low & High & Phosphor screen \\
\hline $\mathrm{CNR}_{\text {Liver }}$ & 1.21 & 1.12 & 1.88 & 1.66 & 1.40 & 1.23 & 2.00 & 1.80 & 0.27 \\
$\mathrm{MTF}_{0.5}$ & 0.41 & 0.55 & 0.33 & 0.46 & 0.49 & 0.58 & 0.39 & 0.48 & 0.37 \\
$\mathrm{MTF}_{1.0}$ & 0.20 & 0.29 & 0.15 & 0.26 & 0.25 & 0.33 & 0.19 & 0.27 & 0.12 \\
\hline \hline
\end{tabular}

the MTF values of the segmented detectors represent the spatial resolution at the center of the detector, where the incident radiation is perpendicular to the detector. The spatial resolution at oblique angles will degrade for detectors with nonfocused geometries. ${ }^{64}$ Due to the limited area of the radiation field $\left(\sim 8.5 \times 3.2 \mathrm{~cm}^{2}\right)$, the CNR results represent radiation incident at very small oblique angles (less than $2^{\circ}$ ) and are not expected to differ greatly from what would be achieved if focused detector geometries were employed. Therefore, it is anticipated that segmented detectors with focused geometries would provide a significantly improved $\mathrm{CNR}$, as well as equivalent, or enhanced spatial resolution performance compared to the phosphor screen detectors used in conventional MV AMFPIs.

It must be emphasized that the present study does not include the simulation of optical transport in the scintillator. The inclusion of optical transport will certainly affect the CNR and MTF performance predicted by radiation transport only. For segmented detectors, if the septal walls are made to be optically opaque, the spatial resolution will not be affected by optical transport, but CNR will degrade due to optical Swank noise, which originates from the depthdependent variation in the x-ray-to-photon conversion gain. An investigation of this noise source using a recently implemented Monte Carlo package (MANTIS) ${ }^{65}$ combining a radiation transport code (PENELOPE-2005) ${ }^{66}$ and an optical transport code (DETECT-II) ${ }^{67}$ is presently underway. It is anticipated that the optical Swank factor can be made relatively high $(>90 \%)$ through optimization of detector designs. If the septal walls are not perfectly opaque, elementto-element optical cross talk will occur, and the spatial resolution of the resulting CT images will degrade. However, the noise level will decrease with increasing optical cross talk due to increased blurring, resulting in an increase in the CNR. For the phosphor screen detector, the inclusion of optical transport would lead to two competing effects on the Noise performance. While a lateral spread of optical photons will result in more statistical averaging in the reconstructed images, thereby reducing Noise, the corresponding optical Swank factor, which is less than unity, will result in an increase in Noise. Therefore, the net effect on CNR performance will be determined by the relative magnitude of these two competing effects.
In summary, the results of this study indicate that MV CBCT systems employing thick segmented scintillating detectors can potentially be used to visualize soft tissue structures at clinically acceptable doses. In order for such detectors to achieve optimum performance for low dose MV CBCT, many design aspects and detector properties will need to be further investigated and optimized, which we believe will be greatly assisted by Monte Carlo simulations of radiation and optical transport.

\section{ACKNOWLEDGMENTS}

The authors would like to thank Dr. Jeffrey Fessler for providing the cone-beam $\mathrm{CT}$ reconstruction algorithm used in this study. The authors also thank Dr. Mahdokht Behravan for her assistance in proofreading. This work was supported by NIH Grant RO1 CA51397.

\section{APPENDIX: ERROR ANALYSIS}

For a Monte Carlo simulation using a given detector and phantom combination, the statistical uncertainty in the signal of voxels in a reconstructed image $(\Delta \mu)$ can be estimated from the statistical uncertainties in the signal of pixels in projection images $(\Delta I)$, using a previously described method. ${ }^{63}$ The Monte Carlo simulation can output the pixel signal $[I(x, y, \theta)]$ and the relative statistical uncertainty $[\Delta I(x, y, \theta) / I(x, y, \theta)]$ associated with that signal for a projection image, where $x$ and $y$ indicate the coordinates of the pixel, and $\theta$ indicates the projection angle. It is found that, in a projection image, the values of $\Delta I(x, y, \theta) / I(x, y, \theta)$ were relatively similar for all pixels in the region of interest. Therefore, a mean value of statistical uncertainty $[\Delta I(\theta) / I(\theta)]$ obtained by averaging $\Delta I(x, y, \theta) / I(x, y, \theta)$ for all pixels in the region of interest, was used to represent the statistical uncertainty for all pixels in this projection image. Also, it is observed that, at all projection angles, the values of $\Delta I(\theta) / I(\theta)$ were almost identical. Therefore, a mean value of statistical uncertainty $(\Delta I / I)$, obtained by averaging $\Delta I(\theta) / I(\theta)$ for all projection angles, was used to represent the statistical uncertainty in the pixel signals in the projection images at all angles for this simulation.

The line integral $(l)$ as shown in Eq. (2), was obtained from the phantom image signal $\left(I_{1}\right)$ and the averaged flood- 
field image signal $\left(I_{0}\right)$. Due to the effect of this averaging, the relative statistical uncertainty in $I_{0}$ is much smaller than that in $I_{1}$. Therefore, the statistical uncertainty in the line integrals $(\Delta l)$ can be estimated ${ }^{68}$ from

$$
\Delta l=\Delta \ln \frac{I_{0}}{I_{1}}=\frac{\Delta\left(I_{0} / I_{1}\right)}{\left(I_{0} / I_{1}\right)}=\sqrt{\left(\frac{\Delta I_{0}}{I_{0}}\right)^{2}+\left(\frac{\Delta I_{1}}{I_{1}}\right)^{2}} \approx \frac{\Delta I_{1}}{I_{1}} .
$$

As shown in Eq. (13) of Ref. 63, the root mean square error of the reconstructed voxel signal $(\Delta \mu)$ can be expressed using the statistical uncertainty in the line integrals:

$$
\Delta \mu=\sqrt{\frac{1.59 \times D}{n \times d^{3}}} \times \Delta l,
$$

where $D$ is the diameter of the reconstructed cylinder $(60.96$ $\mathrm{mm}), d$ is the voxel pitch in the reconstructed image (0.508 $\mathrm{mm}$ ), and $n$ is the number of coplanar paths, which is equal to the product of the number of projections (180) and the number of AMFPI pixels covering the phantom in the scanning direction (128). With these parameter values and using Eq. (A1), Eq. (A2) becomes

$$
\Delta \mu=0.179 \times \frac{\Delta I_{1}}{I_{1}} .
$$

In this study, multiple reconstructed slices were averaged to obtain better statistics. The statistical uncertainty in an averaged slice image $\left(\Delta \mu_{s}\right)$ can be expressed as

$$
\Delta \mu_{s}=\frac{\Delta \mu}{\sqrt{n_{s}}}=\frac{0.179}{\sqrt{n_{s}}} \times \frac{\Delta I_{1}}{I_{1}},
$$

where $n_{s}$ is the number of slices averaged.

In addition, the cupping artifact was removed in the averaged slice image using the correction factors $\mu_{c}(r)$, where $r$ indicates the radial distance from the center of the phantom. The statistical uncertainty in these correction factors $\left[\Delta \mu_{c}(r)\right]$ can be expressed as

$$
\Delta \mu_{c}(r)=\frac{\Delta \mu_{s}}{\sqrt{n_{c}(r)}}=\frac{0.179}{\sqrt{n_{c}(r) \times n_{s}}} \times \frac{\Delta I_{1}}{I_{1}},
$$

where $n_{c}(r)$ is the number of voxels averaged for obtaining the correction factor at a radial distance $r$. In the region selected for evaluation, $n_{c}(r)$ is at least 32 , resulting in values for $\Delta \mu_{c}(r)$ that are much smaller than for $\Delta \mu_{s}$. The statistical uncertainty in the voxel signal after cupping artifact removal $\left(\Delta \mu_{\mathrm{vox}}\right)$ can be estimated from $\Delta \mu_{s}$ and $\Delta \mu_{c}(r)$ :

$$
\Delta \mu_{\mathrm{vox}}=\sqrt{\left(\Delta \mu_{s}\right)^{2}+\left[\Delta \mu_{c}(r)\right]^{2}} \approx \Delta \mu_{s}=\frac{0.179}{\sqrt{n_{s}}} \times \frac{\Delta I_{1}}{I_{1}} .
$$

Therefore, the statistical uncertainties in the mean signal of water background $\left(\Delta \mu_{\text {water }}\right)$ and object $\left(\Delta \mu_{\mathrm{obj}}\right)$ can be expressed as

$$
\Delta \mu_{\mathrm{water}}=\frac{\Delta \mu_{\mathrm{vox}}}{\sqrt{n_{\mathrm{water}}}}=\frac{0.179}{\sqrt{n_{s} \times n_{\mathrm{water}}}} \times \frac{\Delta I_{1}}{I_{1}},
$$

and

$$
\Delta \mu_{\mathrm{obj}}=\frac{\Delta \mu_{\mathrm{vox}}}{\sqrt{n_{\mathrm{obj}}}}=\frac{0.179}{\sqrt{n_{s} \times n_{\mathrm{obj}}}} \times \frac{\Delta I_{1}}{I_{1}},
$$

where $n_{\text {water }}$ and $n_{\text {obj }}$ are the number of voxels in the region of the water background and the object, respectively. Note that $n_{\text {water }}$ is equal to 7756 for the one-object phantom and 4508 for the three-object phantom, while $n_{\text {obj }}$ is equal to 912 for all the examined objects. Moreover, the statistical uncertainty in the standard deviation of the voxel signals in the region of the object $\left(\Delta \sigma_{\text {obj }}\right)$ can be expressed as

$$
\begin{aligned}
\Delta \sigma_{\mathrm{obj}} & =\Delta \sqrt{\frac{\sum_{i=1}^{n_{\text {obj }}}\left(\mu_{i}-\bar{\mu}\right)^{2}}{n_{\mathrm{obj}}-1}} \\
& =\frac{\Delta \mu_{\mathrm{vox}}}{\sqrt{n_{\mathrm{obj}}-1}}=\frac{0.179}{\sqrt{n_{s} \times\left(n_{\mathrm{obj}}-1\right)}} \times \frac{\Delta I_{1}}{I_{1}} .
\end{aligned}
$$

Therefore, the relative error in the Contrast of the object can be expressed as

$$
\frac{\Delta \text { Contrast }}{\text { Contrast }}=\sqrt{\frac{\left(\Delta \mu_{\mathrm{obj}}\right)^{2}+\left(\Delta \mu_{\mathrm{water}}\right)^{2}}{\left(\mu_{\mathrm{obj}}-\mu_{\text {water }}\right)^{2}}+\left(\frac{\Delta \mu_{\mathrm{water}}}{\mu_{\text {water }}}\right)^{2}} .
$$

Furthermore, the relative error in the Noise in the region of the object can be expressed as

$$
\frac{\Delta \text { Noise }}{\text { Noise }}=\sqrt{\left(\frac{\Delta \sigma_{\mathrm{obj}}}{\sigma_{\mathrm{obj}}}\right)^{2}+\left(\frac{\Delta \mu_{\mathrm{water}}}{\mu_{\mathrm{water}}}\right)^{2}} .
$$

Finally, the relative error in the CNR of the object can be expressed as

$$
\frac{\Delta \mathrm{CNR}}{\mathrm{CNR}}=\sqrt{\frac{\left(\Delta \mu_{\mathrm{obj}}\right)^{2}+\left(\Delta \mu_{\mathrm{water}}\right)^{2}}{\left(\mu_{\mathrm{obj}}-\mu_{\mathrm{water}}\right)^{2}}+\left(\frac{\Delta \sigma_{\mathrm{obj}}}{\sigma_{\mathrm{obj}}}\right)^{2}} .
$$

a) Author to whom correspondence should be addressed. Electronic mail: wangyi@umich.edu

${ }^{1} \mathrm{~J}$. A. Purdy, "Advances in three-dimensional treatment planning and conformal dose delivery," Semin. Oncol. 24, 655-671 (1997).

${ }^{2}$ J. A. Purdy, "Intensity-modulated radiation therapy," Int. J. Radiat. Oncol. Biol. Phys. 35, 845-846 (1996).

${ }^{3}$ T. R. Mackie et al., "Image guidance for precise conformal radiotherapy," Int. J. Radiat. Oncol. Biol. Phys. 56, 89-105 (2003).

${ }^{4}$ D. A. Jaffray, D. G. Drake, M. Moreau, A. A. Martinez, and J. W. Wong, "A radiographic and tomographic imaging system integrated into a medical linear accelerator for localization of bone and soft-tissue targets," Int. J. Radiat. Oncol. Biol. Phys. 45, 773-789 (1999).

${ }^{5}$ D. A. Jaffray and J. H. Siewerdsen, "Cone-beam computed tomography with a flat-panel imager: initial performance characterization," Med. Phys. 27, 1311-1323 (2000).

${ }^{6}$ J. H. Siewerdsen and D. A. Jaffray, "Cone-beam computed tomography with a flat-panel imager: magnitude and effects of x-ray scatter," Med. Phys. 28, 220-231 (2001).

${ }^{7}$ D. A. Jaffray, J. H. Siewerdsen, J. W. Wong, and A. A. Martinez, "Flatpanel cone-beam computed tomography for image-guided radiation therapy," Int. J. Radiat. Oncol. Biol. Phys. 53, 1337-1349 (2002).

${ }^{8}$ B. A. Groh, J. H. Siewerdsen, D. G. Drake, J. W. Wong, and D. A. Jaffray, "A performance comparison of flat-panel imager-based MV and kV cone-beam CT,” Med. Phys. 29, 967-975 (2002). 
${ }^{9}$ S. A. Graham, D. J. Moseley, J. H. Siewerdsen, and D. A. Jaffray, "Compensators for dose and scatter management in cone-beam computed tomography," Med. Phys. 34, 2691-2703 (2007).

${ }^{10} \mathrm{U}$. Oelfke et al., "Linac-integrated kV-cone beam CT: technical features and first applications," Med. Dosim. 31, 62-70 (2006).

${ }^{11}$ D. Letourneau et al., "Cone-beam-CT guided radiation therapy: technical implementation," Radiother. Oncol. 75, 279-286 (2005).

${ }^{12}$ J. L. Barker, Jr. et al., "Quantification of volumetric and geometric changes occurring during fractionated radiotherapy for head-and-neck cancer using an integrated CT/linear accelerator system," Int. J. Radiat. Oncol. Biol. Phys. 59, 960-970 (2004).

${ }^{13} \mathrm{~K}$. Kuriyama et al., "A new irradiation unit constructed of self-moving gantry-CT and linac," Int. J. Radiat. Oncol. Biol. Phys. 55, 428-435 (2003).

${ }^{14} \mathrm{~J}$. M. Balter et al., "Daily targeting of intrahepatic tumors for radiotherapy," Int. J. Radiat. Oncol. Biol. Phys. 52, 266-271 (2002).

${ }^{15}$ J. Lattanzi, S. McNeeley, A. Hanlon, T. E. Schultheiss, and G. E. Hanks, "Ultrasound-based stereotactic guidance of precision conformal external beam radiation therapy in clinically localized prostate cancer," Urology 55, 73-78 (2000).

${ }^{16} \mathrm{~K}$. M. Langen et al., "The use of megavoltage CT (MVCT) images for dose recomputations," Phys. Med. Biol. 50, 4259-4276 (2005).

${ }^{17}$ S. L. Meeks et al., "Ultrasound-guided extracranial radiosurgery: Technique and application,” Int. J. Radiat. Oncol. Biol. Phys. 55, 1092-1101 (2003).

${ }^{18}$ R. G. Simpson, C. T. Chen, E. A. Grubbs, and W. Swindell, "A 4-MV CT scanner for radiation therapy: The prototype system," Med. Phys. 9, 574579 (1982).

${ }^{19}$ W. Swindell, R. G. Simpson, J. R. Oleson, C. Chen, and E. A. Grubbs, "Computed tomography with a linear accelerator with radiotherapy applications," Med. Phys. 10, 416-420 (1983).

${ }^{20}$ M. A. Mosleh-Shirazi, P. M. Evans, W. Swindell, S. Webb, and M. Partridge, "A cone-beam megavoltage CT scanner for treatment verification in conformal radiotherapy," Radiother. Oncol. 48, 319-328 (1998).

${ }^{21} \mathrm{~J}$. Pouliot et al., "Low-dose megavoltage cone-beam CT for radiation therapy,” Int. J. Radiat. Oncol. Biol. Phys. 61, 552-560 (2005).

${ }^{22}$ O. Morin et al., "Megavoltage cone-beam CT: System description and clinical applications," Med. Dosw. 31, 51-61 (2006).

${ }^{23} \mathrm{O}$. Morin et al., "Patient dose considerations for routine megavoltage cone-beam CT imaging," Med. Phys. 34, 1819-1827 (2007).

${ }^{24}$ E. C. Ford et al., "Cone-beam CT with megavoltage beams and an amorphous silicon electronic portal imaging device: potential for verification of radiotherapy of lung cancer," Med. Phys. 29, 2913-2924 (2002).

${ }^{25}$ E. J. Seppi et al., "Megavoltage cone-beam computed tomography using a high-efficiency image receptor," Int. J. Radiot. Biol. Phys. 55, 793-803 (2003).

${ }^{26}$ J. Chang et al., "Integrating respiratory gating into a megavoltage conebeam CT system," Med. Phys. 33, 2354-2361 (2006).

${ }^{27}$ J. Sillanpaa et al., "Developments in megavoltage cone beam CT with an amorphous silicon EPID: Reduction of exposure and synchronization with respiratory gating," Med. Phys. 32, 819-829 (2005).

${ }^{28}$ J. Sillanpaa et al., "Low-dose megavoltage cone-beam computed tomography for lung tumors using a high-efficiency image receptor," Med. Phys. 33, 3489-3497 (2006).

${ }^{29}$ E. K. Hansen et al., "Image-guided radiotherapy using megavoltage conebeam computed tomography for treatment of paraspinous tumors in the presence of orthopedic hardware," Int. J. Radiat. Oncol. Biol. Phys. 66, 323-326 (2006).

${ }^{30}$ F. A. Ghelmansarai et al., "Soft tissue visualization using a highly efficient megavoltage cone beam CT imaging system," Proc. SPIE 5745, 159-170 (2005).

${ }^{31}$ S. Midgley, R. M. Millar, and J. Dudson, "A feasibility study for megavoltage cone beam CT using a commercial EPID," Phys. Med. Biol. 43, 155-169 (1998).

${ }^{32}$ G. Pang, X. Mei, and J. A. Rowlands, "Development of a novel high quantum efficiency flat panel detector for megavoltage cone beam CT/ DT: Construction and evaluation of a prototype single-row detector," Med. Phys. 33, 2281 (2006).

${ }^{33} \mathrm{H}$. Keller et al., "Monte Carlo study of a highly efficient gas ionization detector for megavoltage imaging and image-guided radiotherapy," Med. Phys. 29, 165-175 (2002).

${ }^{34} \mathrm{H}$. Amols, D. A. Jaffray, and C. G. Orton, "Image-guided radiotherapy is being overvalued as a clinical tool in radiation oncology," Med. Phys. 33,
3583-3586 (2006).

${ }^{35}$ O. Morin et al., "Dose calculation using megavoltage cone beam CT imaging," Int. J. Radiat. Oncol. Biol. Phys. 63, S62-S63 (2005).

${ }^{36}$ H. Guan, F. F. Yin, and J. H. Kim, "Accuracy of inhomogeneity correction in photon radiotherapy from CT scans with different settings," Phys. Med. Biol. 47, N223-N231 (2002).

${ }^{37}$ F. F. Yin, H. Guan, and W. Lu, "A technique for on-board CT reconstruction using both kilovoltage and megavoltage beam projections for 3D treatment verification," Med. Phys. 32, 2819-2826 (2005).

${ }^{38}$ Y. El-Mohri, K.-W. Jee, L. E. Antonuk, M. Maolinbay, and Q. Zhao, "Determination of the detective quantum efficiency of a prototype, megavoltage indirect detection, active matrix flat-panel imager," Med. Phys. 28, 2538-2550 (2001).

${ }^{39}$ L. E. Antonuk, "Electronic portal imaging devices: A review and historical perspective of contemporary technologies and research," Phys. Med. Biol. 47, N31-N65 (2002).

${ }^{40} \mathrm{G}$. Pang and J. A. Rowlands, "Development of high quantum efficiency, flat panel, thick detectors for megavoltage x-ray imaging: A novel directconversion design and its feasibility," Med. Phys. 31, 3004-3016 (2004).

${ }^{41}$ E. J. Morton, W. Swindell, D. G. Lewis, and P. M. Evans, "A linear array, scintillation crystal-photodiode detector for megavoltage imaging," Med. Phys. 18, 681-691 (1991).

${ }^{42}$ D. G. Lewis, W. Swindell, E. J. Morton, P. M. Evans, and Z. R. Xiao, “A megavoltage CT scanner for radiotherapy verification," Phys. Med. Biol. 37, 1985-1999 (1992).

${ }^{43} \mathrm{~K}$. Nakagawa et al., "Real-time beam monitoring in dynamic conformation therapy," Int. J. Radiat. Oncol. Biol. Phys. 30, 1233-1238 (1994).

${ }^{44}$ S. Rathee, D. Tu, T. T. Monajemi, D. W. Rickey, and B. G. Fallone, "A bench-top megavoltage fan-beam $\mathrm{CT}$ using $\mathrm{CdWO}_{4}$-photodiode detectors. I. System description and detector characterization," Med. Phys. 33, 1078-1089 (2006).

${ }^{45}$ T. T. Monajemi, D. Tu, B. G. Fallone, and S. Rathee, "A bench-top megavoltage fan-beam $\mathrm{CT}$ using $\mathrm{CdWO}_{4}$-photodiode detectors. II. Image performance evaluation," Med. Phys. 33, 1090-1100 (2006).

${ }^{46}$ M. A. Mosleh-Shirazi, W. Swindell, and P. M. Evans, "Optimization of the scintillation detector in a combined 3D megavoltage CT scanner and portal imager," Med. Phys. 25, 1880-1890 (1998).

${ }^{47}$ M. A. Mosleh-Shirazi et al., "Rapid portal imaging with a high-efficiency, large field-of-view detector," Med. Phys. 25, 2333-2346 (1998).

${ }^{48}$ X. Mei, J. A. Rowlands, and G. Pang, "Electronic portal imaging based on Cerenkov radiation: A new approach and its feasibility," Med. Phys. 33, 4258-4270 (2006).

${ }^{49}$ O. Z. Ostapiak, P. F. O’Brien, and B. A. Faddegon, "Megavoltage imaging with low $Z$ targets: Implementation and characterization of an investigational system," Med. Phys. 25, 1910-1918 (1998).

${ }^{50} \mathrm{~A}$. Sawant et al., "Segmented crystalline scintillators: An initial investigation of high quantum efficiency detectors for megavoltage x-ray imaging," Med. Phys. 32, 3067-3083 (2005).

${ }^{51}$ A. Sawant et al., "Segmented crystalline scintillators: Empirical and theoretical investigation of a high quantum efficiency EPID based on an initial engineering prototype CsI(Tl) detector," Med. Phys. 33, 10531066 (2006).

${ }^{52}$ D. Sheikh-Bagheri, "Monte Carlo Study of Photon Beams from Medical Linear Accelerators: Optimization, Benchmark, and Spectra," Ph. D. thesis, Department of Physics, Carleton University (1999).

${ }^{53}$ M. K. Yu, R. S. Sloboda, and B. Murray, "Linear accelerator photon beam quality at off-axis points," Med. Phys. 24, 233-239 (1997).

${ }^{54}$ L. E. Antonuk et al., "Initial performance evaluation of an indirectdetection, active matrix flat-panel imager (AMFPI) prototype for megavoltage imaging,” Int. J. Radiat. Oncol. Biol. Phys. 42, 437-454 (1998).

${ }^{55}$ Data sheet of tissue characterization phantom (Gammex 467), Gammex RMI, Inc., Wisconsin (2006).

${ }^{56}$ International Commission on Radiation Units and Measurements (ICRU) Report 46, "Photon, Electron, Proton and Neutron Interaction Data for Body Tissues," ICRU Publications, Bethesda, Maryland (1992).

${ }^{57}$ I. Kawrakow and D. W. O. Rogers, "The EGSnrc Code System: Monte Carlo Simulation of Electron and Photon Transport," Technical Report PIRS-701, National Research Council of Canada, Ottawa, Canada (2000).

${ }^{58}$ J. A. Treurniet, B. R. B. Walters, and D. W. O. Rogers, "BEAMnrc, DOSXYZnrc, and BEAMDP GUI User's Manual," NRC Report PIRS 0623, National Research Council of Canada, Ottawa, Canada (2001).

${ }^{59} \mathrm{G}$. H. Glover, "Compton scatter effects in CT reconstructions," Med. 
Phys. 9, 860-867 (1982).

${ }^{60} \mathrm{R}$. Ning et al., "Flat panel detector-based cone-beam volume CT angiography imaging: system evaluation," IEEE Trans. Med. Imaging 19, 949 963 (2000).

${ }^{61}$ M. Kachelriess, K. Sourbelle, and W. A. Kalender, "Empirical cupping correction: a first-order raw data precorrection for cone-beam computed tomography," Med. Phys. 33, 1269-1274 (2006).

${ }^{62}$ L. A. Feldkamp, L. C. Davis, and J. W. Kress, "Practical cone-beam algorithm," J. Opt. Soc. Am. A 1, 612-619 (1984).

${ }^{63}$ R. H. Huesman, "The effects of a finite number of projection angles and finite lateral sampling of projections on the propagation of statistical errors in transverse section reconstruction," Phys. Med. Biol. 22, 511-521 (1977).

${ }^{64}$ T. T. Monajemi, B. G. Fallone, and S. Rathee, "Thick, segmented $\mathrm{CdWO}_{4}$-photodiode detector for cone beam megavoltage CT: A Monte
Carlo study of system design parameters," Med. Phys. 33, 4567-4577 (2006).

${ }^{65}$ A. Badano and J. Sempau, "MANTIS: combined x-ray, electron and optical Monte Carlo simulations of indirect radiation imaging systems," Phys. Med. Biol. 51, 1545-1561 (2006).

${ }^{66}$ F. Salvat, J. M. Fernández-Varea, and J. Sempau, PENELOPE, A Code System for Monte Carlo Simulation of Electron and Photon Transport (Issy-les-Moulineaux, France, 2003).

${ }^{67} \mathrm{~A}$. Badano, "Image quality degradation by light scattering processes in high performance display devices for medical imaging," Ph.D. thesis, Center for Integrated Microsystems, University of Michigan (1999).

${ }^{68}$ J. R. Taylor, An Introduction to Error Analysis: The Study of Uncertainties in Physical Measurements, 2nd ed. (University Science Books, California, 1997). 